\title{
Light Source Unit for Hybrid Photodiode Calibration
}

\author{
J.Freeman, S.Los and A.Ronzhin \\ Fermi National Accelerator Laboratory \\ P.O. Box 500, Batavia, Illinois 60510
}

\section{INTRODUCTION}

The hybrid photodiode (HPD [1]) will be used for the CMS HCAL readout [2]. The light source was designed and built to check the functionality of the readout. The technical parameters of the unit are presented.

\section{CALIBRATION SYSTEM REQUIREMENTS FOR CMS}

The goal of this work was to design and make a prototype light calibration unit for the CMS HCAL. The light produced by the unit has to cover the full HCAL dynamic range and to be short in time. The Large Hadron Collider (LHC) will operate with a 25 ns bunch period. The HCAL's optical signal for hadron showers has a rise time of $10 \mathrm{~ns}$ and a fall time of $20 \mathrm{~ns}$, with $90 \%$ of the integrated signal in $75 \mathrm{~ns}$. The light yield of the HCAL is between 10 and 20 photoelectrons per $\mathrm{GeV}$, and the dynamic range spans from a single photoelectron to 70000 photoelectrons $(3.5 \mathrm{TeV})$. Stability of the unit's parameters is important. Some issues are discussed below:

The HCAL uses Kuraray SCSN 81 blue scintillator. This light is converted to green by Kuraray Y11 waveshifting fiber inserted in the scintillators and transported to the HPD's by clear optical fibers. This means that the calibration unit should provide green light for the HPD's calibration. The calibration laser is a $\mathrm{N}_{2}$ laser, with light emission at $337 \mathrm{~nm}$. This light needs to be shifted into the blue so that the wave-shifting fibers can absorb and re-emit it as green. On the other hand, the transparent mixer containing the green fibers should not scintillate. Reasons for this are reduce sensitivity to particles passing through the mixer, ageing, etc. A search was made for materials that will absorb UV light and re-emit it in the blue wavelengths. Ultimately, the decision was made to use acrylic plastic for the mixer, and a very small piece of scintillator at the output of the laser quartz fiber to shift the UV into blue. The small volume of the scintillator thus reduces the likelihood that particles will hit it and give spurious light into the HCAL during operation. 
LED's are not generally used as a form of precision monitoring. This is due to factors including aging, temperature dependent light yield, and radiation damage. In addition, a system based on LED's would require a large number of them placed in each readout compartment. The differing properties of each LED would then need to be monitored. It is difficult to maintain stability of LED's being operated at very low light levels ( 1 - few photoelectrons). Since the LED's are mounted on the detector, maintenance and replacement is difficult.

On the other hand, using lasers for precision monitoring is a well-established technique. The laser system has the advantage that since it is so much brighter than LED's, a single laser can be used to calibrate the entire detector. The laser light can be transported from the control room down to the experiment. Thus, the laser can be placed in a well-controlled environment, away from radiation. Since a single laser is used in the calibration, monitoring of the light source becomes much easier. Lasers can easily span the required dynamic range by use of neutral density filters. Consequently we have chosen a laser system to do the primary monitoring of the detector. The laser system will be used to monitor the HPD stability (1\% accuracy) during data taking.

The LED system has the advantages of simplicity, portability, and convenience. The LED system is especially useful during setup, installation, and assembly. It is also will be in operation during the experiment for quick testing. The LED system will be very useful to check functionality of the HCAL readout (HPD's, electronics, HV, LV, interconnections, cables, contacts, etc.), especially in the factory and test beams.

\section{PRELIMINARY LED TEST RESULTS}

The following types of the LED's were chosen for study: Hewlett Packard blue (HLMP-CB15), Toshiba blue (catalog \#25-365 (C)), Nichia blue (NSPB500S) and Nichia green (NSPG500S) LED's. The choice was done according to good timing and brightness of the LED's. The timing of the LED's was studied first. The PIN diode Hamamatsu S3883 with $1.5 \mathrm{~mm}$ diameter sensitive area was used as photodetector because of very fast rise and fall time ( $1 \mathrm{~ns})$. The PIN diode electrical scheme is shown in fig. 1 . AV$1000-\mathrm{C}, \mathrm{HP}$ and current generator were in use as the LED's drivers. Electrical signal line shapes were measured using a Tektronics TDS $3054500 \mathrm{MHz}$ digital oscilloscope. Figures 2, 3 show PIN diode responses under direct LED's illumination. The LED's driver in the case is a voltage generator. The Toshiba blue and Nichia green LED's were selected for additional study because they have better timing properties.

\section{LASER SETUP}

A nitrogen laser with $337 \mathrm{~nm}$ of wavelength was used. Maximum power per pulse is $400 \mathrm{mkJ}$. Maximum of frequency is $20 \mathrm{~Hz}$. The setup contains laser light splitter made of 31 quartz fibers which uniformly capture and transport the laser light. Each fiber is 5 meters long and $0.6 \mathrm{~mm}$ of diameter. A mechanical shutter installed before quartz fiber splitter was used to change the laser light intensity. 


\section{UNIT DESCRIPTION}

The basic concept of the calibration unit is that light (UV from the laser, green and blue from LED's) is mixed in a light mixer. Waveshifting green fiber optically coupled to the mixer captures the light and transports it to the HPD pixels. Photodiodes optically coupled to the light mixer monitor the light signals.

The sketch of the unit is shown in fig. 4. Four optical connectors, four LEMO connectors and one SMA connector were installed on the front panel of the unit. Each optical connector contains 18 fiber of $0.94 \mathrm{~mm}$ diameter installed inline with a $1.41 \mathrm{~mm}$ step. The number of the fibers is sufficient to illuminate simultaneously one 73 pixel HPD or four 19 pixel HPD's. Two LEMO front panel connectors are used for LED drivers. Another two serve for PIN diode readout. The SMA connector permits laser light input. A transparent acrylic plastic mixer of $13 \mathrm{~mm}$ diameter and $37 \mathrm{~mm}$ of length is placed inside of the unit.

Two versions of optical coupling of the LED's to the light mixer were tested. In the first version, two holes (for separate blue and green LED's) were drilled into one side of the mixer (version 1, fig.5). For version 2, a single hole in the geometrical center of the end of the mixer was drilled. fig.6). The cables for the LED's were double shielded inside of the unit to suppress pickup noise in the photodiodes. For either version of the light mixer, a hole of $10 \mathrm{~mm}$ diameter and depth was drilled in the face opposite the LED's for placement of the 72 waveshifting green fibers. The fibers were $0.94 \mathrm{~mm}$ in diameter and about $120 \mathrm{~mm}$ long, of type KURARAY, Y11. A $0.6 \mathrm{~mm}$ diameter quartz fiber was used to input the laser light. The fiber was terminated into an SMA connector on the front panel of the unit. The hole of $1.5 \mathrm{~mm}$ diameter was drilled into the mixer from the LED side for SCSN 81 scintillator and the fiber insertion into the mixer. A small piece of the scintillator (1.4 mm diameter, $3-7 \mathrm{~mm}$ of length) was inserted first, followed by the quartz fiber. Two PIN diodes (1.5 mm diameter S3883, Hamamatsu and $2.5 \mathrm{~mm}$ diameter of sensitive area, EG\&G) were situated by the LED's. The fibers, PIN diodes, and LED's are glued to the mixer with 5 minute optical epoxy. The PIN diode circuit diagram is the same as shown in fig. 1. A battery was used for the power supply of the PIN diodes. The optical mixer is wrapped in white paper. The mixer and the battery are attached to the unit body by spring and bolt.

\section{MEASUREMENTS}

The light was carried from the calibration unit to the HPD unit by a $400 \mathrm{~mm}$ long optical cable consisting of $180.94 \mathrm{~mm}$ diameter clear fibers. At the HPD unit, the light was transported to the individual pixels of the HPD by an additional $100 \mathrm{~mm}$ of clear $0.94 \mathrm{~mm}$ diameter fiber. The test setup was: a voltage pulse was injected into the LED's using a AV-1000-C pulse generator; the electrical signal from the HPD was amplified using a charge integrating amplifier with a time constant about $250 \mathrm{~ns}$ and amplification about 450 . This amplifier was originally designed for the D0 experiment in part of the muon detector system [3]. Electrical signal line shapes were measured using a Tektronics TDS $3054500 \mathrm{MHz}$ digital oscilloscope. A 19 pixel HPD was used for the tests. 
When working with the laser, the light was transported to the unit by an optical quartz fiber terminated by SMA connector.

The quantities measured were the number of photoelectrons, the uniformity of the light level from 72 light output of the unit and the shape of the HPD signals from the pixels.

The D0 amplifiers were used to measure light provided by the unit in number of photoelectron's and the uniformity of the light for different light output of the unit. An amplitude of $1 \mathrm{~V} / 50 \mathrm{Ohm}$ of the signal corresponds approximately to 10,000 photoelectrons taking into account the HPD gain and the gain of the amplifier. No change of the D0 amplifiers output signal shape was observed for the full dynamic range under different measurement conditions. The shape of the signal is shown in fig. 7 . The number of photoelectrons and the light uniformity of the unit were measured by oscilloscope and by a DAQ based on the ADC CAEN C205 (32 channels per module).

The 17 D0 amplifiers and 17 pixels of the HPD used to estimate the level of the nonuniformity for different light out of the calibration unit. The spread of the amplifiers, pixels and ADC gain is no more than $+/-5 \%$ in sum. PIN diode signals were taken to normalize data for the full dynamic range especially when working with the laser.

The shape of the HPD signal was measured by oscilloscope. The data will be presented for the central pixel of the 19 pixels HPD because the HPD keeps the same shape for other pixels.

The data obtained with LED's is shown for different LED placement into the mixer (figs. 5, 6). The laser data will be presented next because these are the same for both versions. The number of photoelectrons is in the range $10-10,000$ for each light channel, and controlled by the AV-1000-C driver amplitude and pulse duration. Maximum amplitude is $10 \mathrm{~V} / 50 \mathrm{Ohm}$ and driver pulse duration is $30 \mathrm{~ns}$. This range is about $10-100,000$ for the green LED. The corresponding driver pulses are shown in fig. 8 . The light uniformity is better for central LED positioning and is about $+/-10 \%$ (version 2 , fig. 9). It is about $+/-30 \%$ when LED displaced from the center of the mixer (version 1).

An example of the HPD signal shape is presented in fig. 10. The shape is the same while firing green or blue LED for both versions. All timing data are taken under $10 \mathrm{kV}$ of high voltage and $100 \mathrm{~V}$ bias voltage of the HPD if not specifically determined. It is worth noting that the shape can be changed by adjustment of LED's driver parameters.

The number of photoelectrons under HPD laser illumination is in the range $10-$ $1,000,000$ per channel. Note the HPD signal starts to saturate when the number of the photoelectrons is more than $200,000 / \mathrm{mm}^{2}$ of Si per $10 \mathrm{~ns}$. An example of the HPD signal shapes are shown in fig. 11 . This shape is also can be changed by some change of the optical elements of the unit. This will be discussed below. The nonuniformity of the different light output of the unit is also about $+/-10 \%$ when illuminating by the laser (fig. 12). The data taken by oscilloscope and by the DAQ are in good agreement. 


\section{DISCUSSION}

Let's start with the laser data. The shapes of the HPD signal under different conditions are presented in fig. 13. The setup was: laser, 5 meters quartz fiber, HPD, oscilloscope. The shape of the signal can be changed if $15 \mathrm{~mm}$ acrylic plastic or $3 \mathrm{~mm}$ SCSN81 scintillator is added just before the HPD input window. The HPD signal is sharper if $30 \mathrm{~mm}$ SCSN81 is added before the HPD (fig. 14). The bias voltage dependence of the HPD signal shape is also presented in the same figure 14.

The effect relates with the UV light reemitted by SCSN81 or acrylic and captured by the HPD. Birks investigated such effects in the early 1950's. The UV light absorption and re-emittance in transparent materials are very well described in literature. The mixer can be manufactured from polystyrene to get a sharper pulse shape. We did not study the effects carefully and empirically installed $5 \mathrm{~mm}$ of the SCSN81 in our acrylic mixer to get pulse shape adequate to our requirements. Note that the HPD pulse shape does not change noticeably in the dynamic range about 100 that is possible in the direct measurements.

Light nonuniformity of the unit should be about $+/-10 \%$. This light spread is acceptable from test beam experience. The level is achievable in the design for laser and central LED positioning. A longer mixer or closer LED's positioning to the center of the mixer (version 1) are also possible. Note that mixer's wrapping by white paper improves uniformity a little. The light nonuniformity in the version 2 of the design can be improved by placing semitransparent light scattering material between LED/laser and the green fibers. The price for this is more mechanical work.

\section{UNIT PERFORMANCE.}

The light yield obtained in the unit is more than enough for both LED and laser illumination. The HPD pulse shapes measured are compatible with CMS HCAL requirements for both laser and LED. The light nonuniformity of the unit is appropriate for the HPD calibration requirements.

\section{RESULTS}

The light source unit for the HPD calibration is designed, manufactured, and tested. The parameters of the unit fit the calibration requirements. 
SPECIFICATIONS OF THE UNIT

Size

Weight

LEMO IN for LED

LEMO OUT for PIN

SMA for laser IN

Light OUT connector

Number of light channels

Green fiber diameter

Quartz fiber diameter

LED's

Blue LED dynamic range

(versions 1,2)

Driver pulse

Green LED dynamic range

(versions 1,2)

Driver pulse

Current LED's limit

Frequency

LASER

Laser

Max. power per pulse

Dynamic range

Long term stability

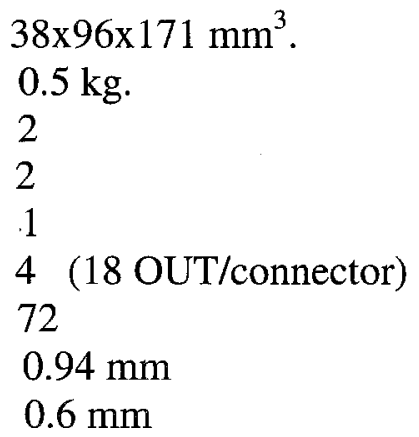

$10-10,000$ photoelectrons/per light channel.

10 - $30 \mathrm{ns,}$ maximum amplitude $10 \mathrm{~V} / 50 \mathrm{Ohm}$.

10 - 100,000 photoelectrons/per light/channel.

$10-30 \mathrm{~ns}$, maximum amplitude $10 \mathrm{~V} / 50 \mathrm{Ohm}$.

$10 \mathrm{~mA}$

up to $1 \mathrm{MHz} / 10 \mathrm{~mA}$ current limit

$\mathrm{N}_{2}, 337 \mathrm{~nm}$.

$400 \mathrm{mkJ}$

$1-1,000,000$ photoelectrons/per light channel. not studied yet.

\section{CONCLUSION}

1. New HPD calibration unit fits well mechanically into the last version of the miniboxes, and into the readout box (RBX, barrel part of HCAL).

2. The unit provides 3000 times more light than a previous calibration module with the same driver pulse.

3. The new unit works with standard optical cables approved for HCAL optical readout. Different parts of the HCAL optical system (optical decoder unit (ODU) optical channels, optical cables, etc.) can be tested with the unit. 
Figures.

1. Electrical diagram PIN diode scheme.

2. PIN diode S3883 Hamamatsu response for Toshiba blue LED.

3. PIN diode S3883 Hamamatsu response for Nichia green LED.

4. Sketch of the unit.

5. Sketch of mixer, version 1.

6. Sketch of mixer, versions 2 .

7. Trace is for D0 charge integrating amplifier.

8. Traces are for pulses driving the green LED.

9. Histograms are for the mean charge value D0 amplifiers measured for the HPD pixels under green LED illumination ( $a, b, c, d$ are for 4 light out of the unit).

10. Trace is for HPD, green LED.

11. Traces are for HPD ( $\mathrm{a}$ - laser, quartz fiber, $3 \mathrm{~mm}$ SCSN81 just before HPD, b quartz fiber of the laser is hooked up to calibration unit).

12. Histograms are for the mean charge value D0 amplifiers measured for the HPD pixels under laser illumination ( $a, b, c, d$ are for 4 light out of the unit).

13. Traces are for HPD illuminated by laser through 5 meters quartz fiber (a - direct illumination, $b-3 \mathrm{~mm}$ SCSN81 before HPD, $\mathrm{c}-15 \mathrm{~mm}$ of acrylic plastic before HPD).

14. The same as 13 , but $30 \mathrm{~mm}$ SCSN81 before HPD (a, b, c, d, e, f - for bias voltage in the range $50-100$ Volts. 
Bibliography

1. The Hybrid Photodiode tubes (HPD), Catalog of DEP DELFT INSTRUMENTS.

2. CMS The Hadron Calorimeter Project. Technical Design Report. CERN/LHCC 97-31, CMS TDR 2, 20 June 1997.

3. D0 charge integrating amplifier, data given by Sten Hansen and Claudio Rivetta, Fermilab. 


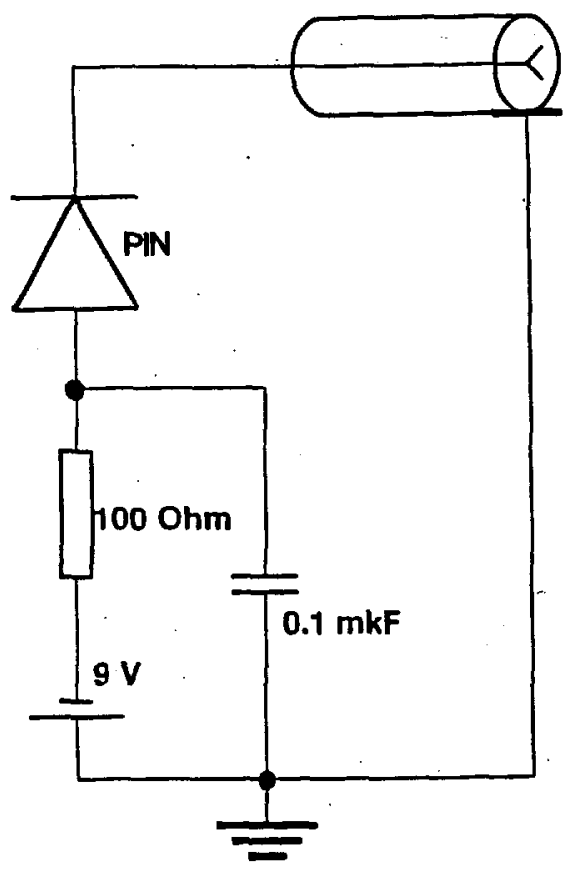

Fig.1

TOSHIBA 6 Cd Blue LED Light Output with a Voltage Pulser (OPA633) and a 1 mm2 PIN Diod

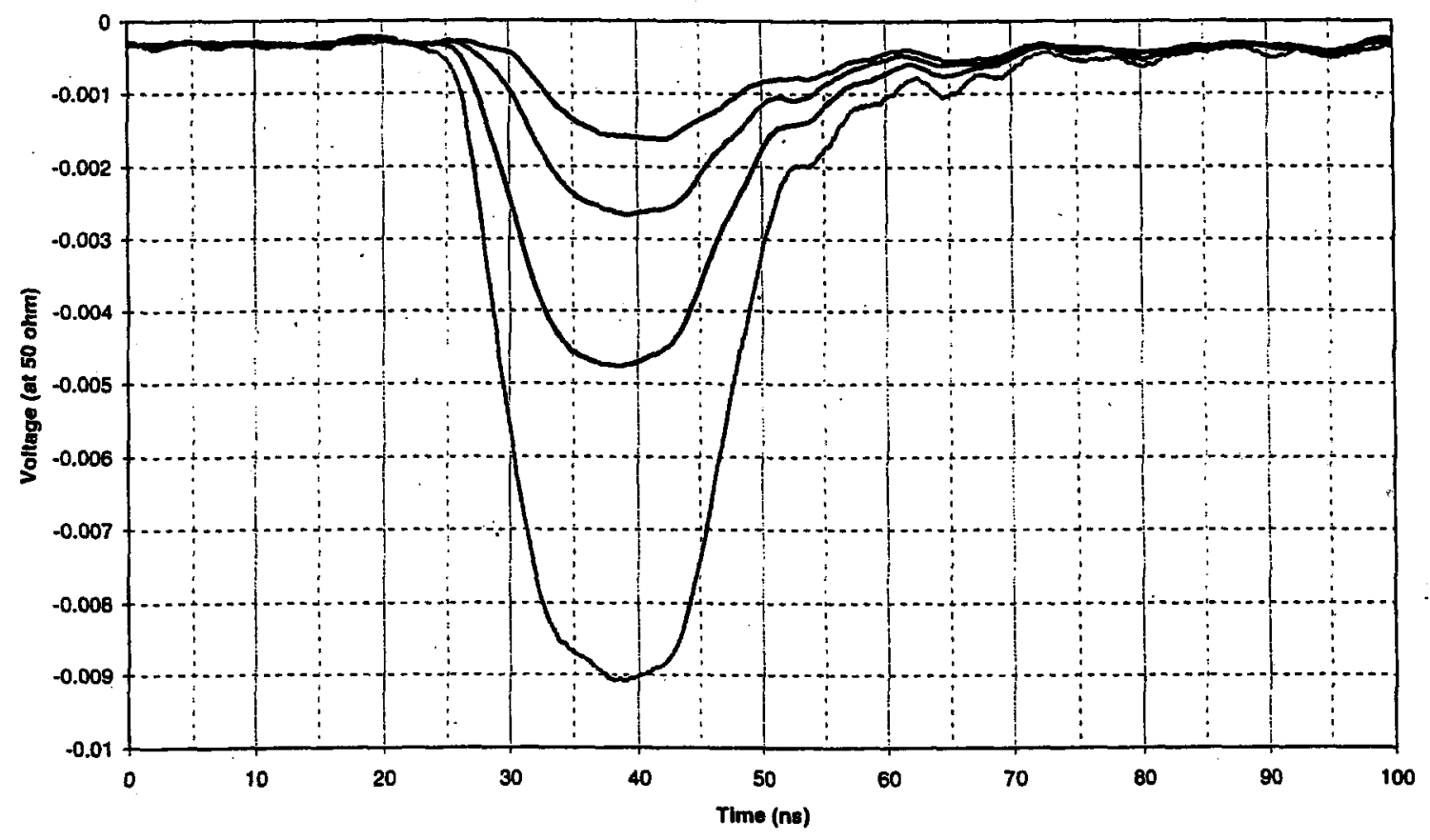

Fig.2 
Nichia 10 Cd Green LED Light Output

with a Voltage Pulser (OPA633) and a $1 \mathrm{~mm} 2$ PIN Diod

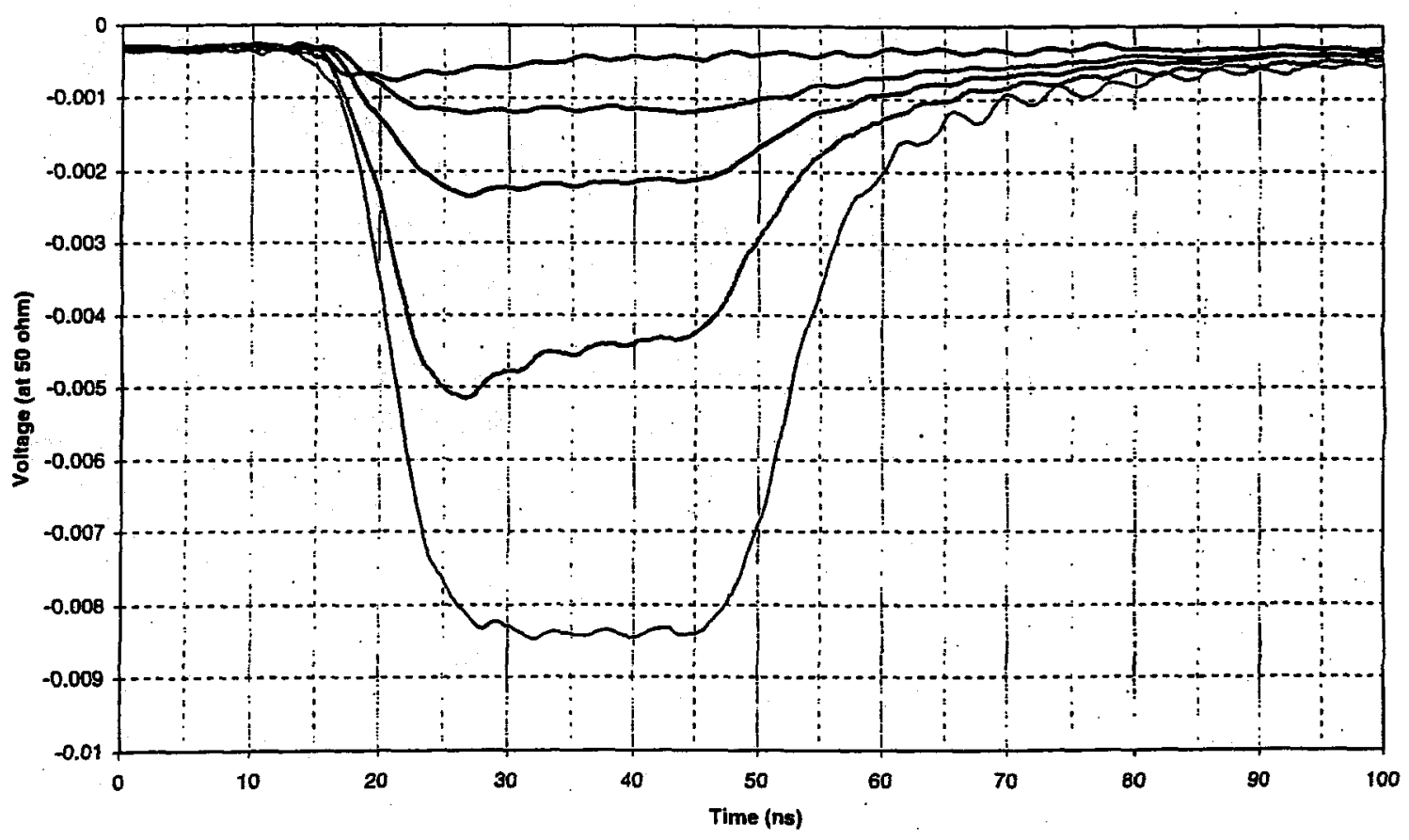

Fig.3

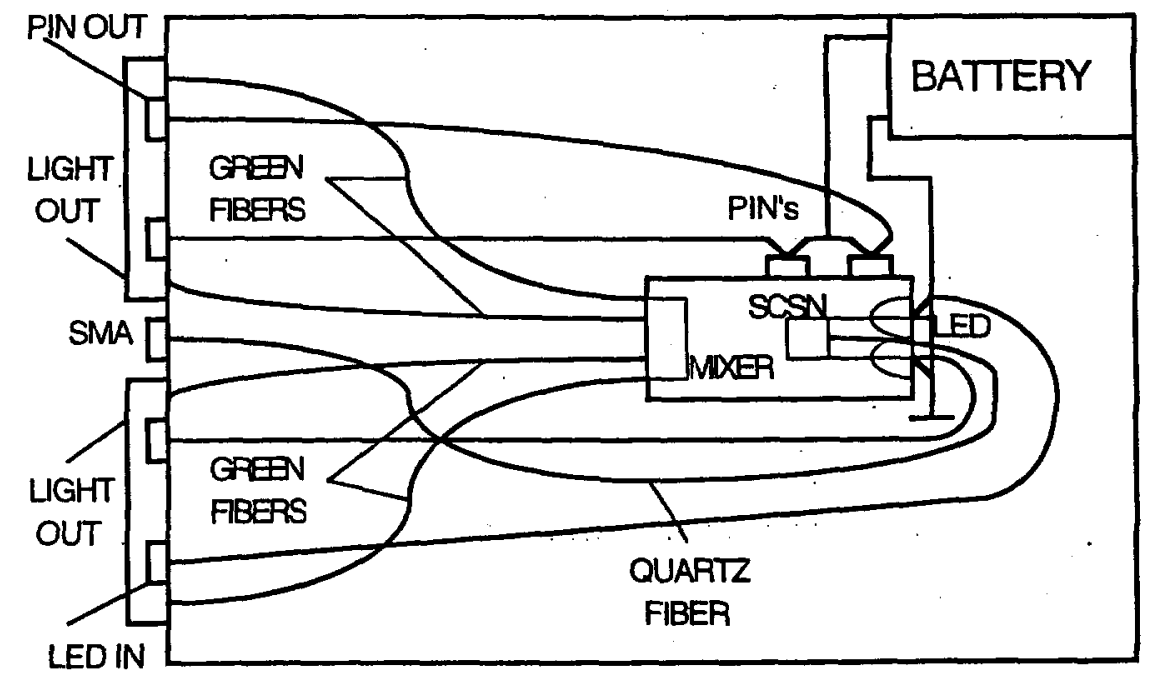

Fig.4 


\section{LIGHT MIXER, 1}

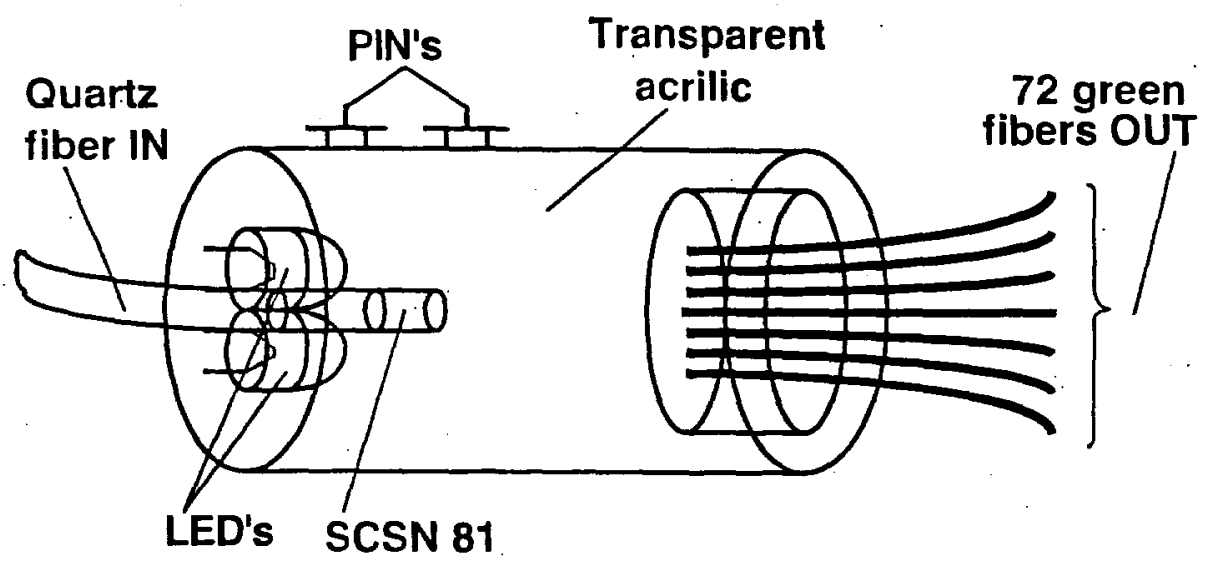

Fig.5

\section{LIGHT MIXER, 2}

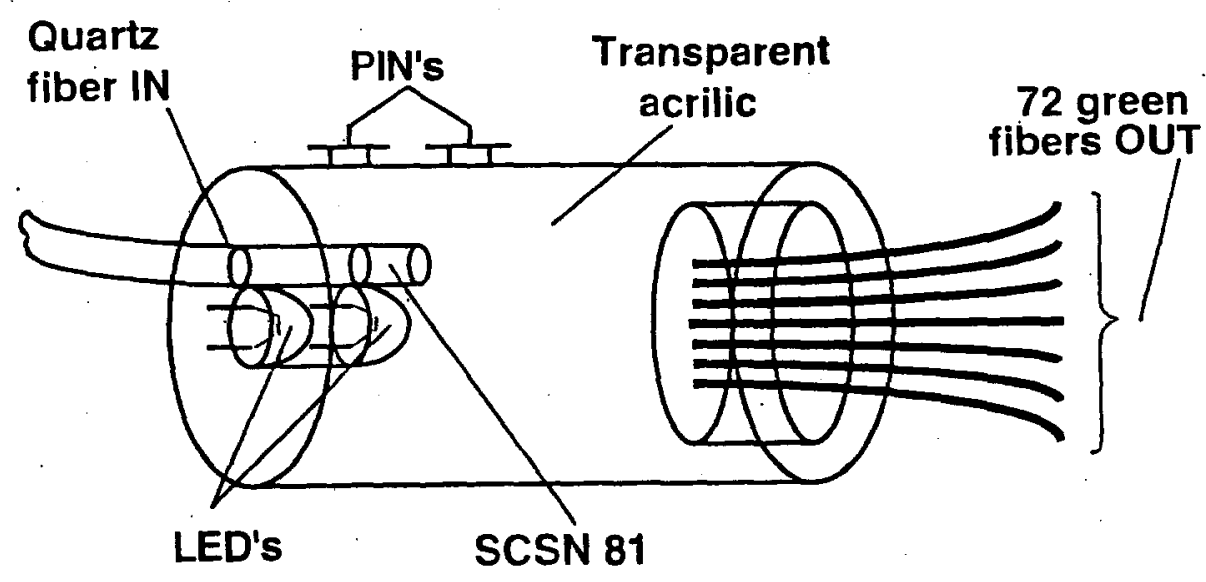

Fig6 


\section{D0, 5,000 , laser}

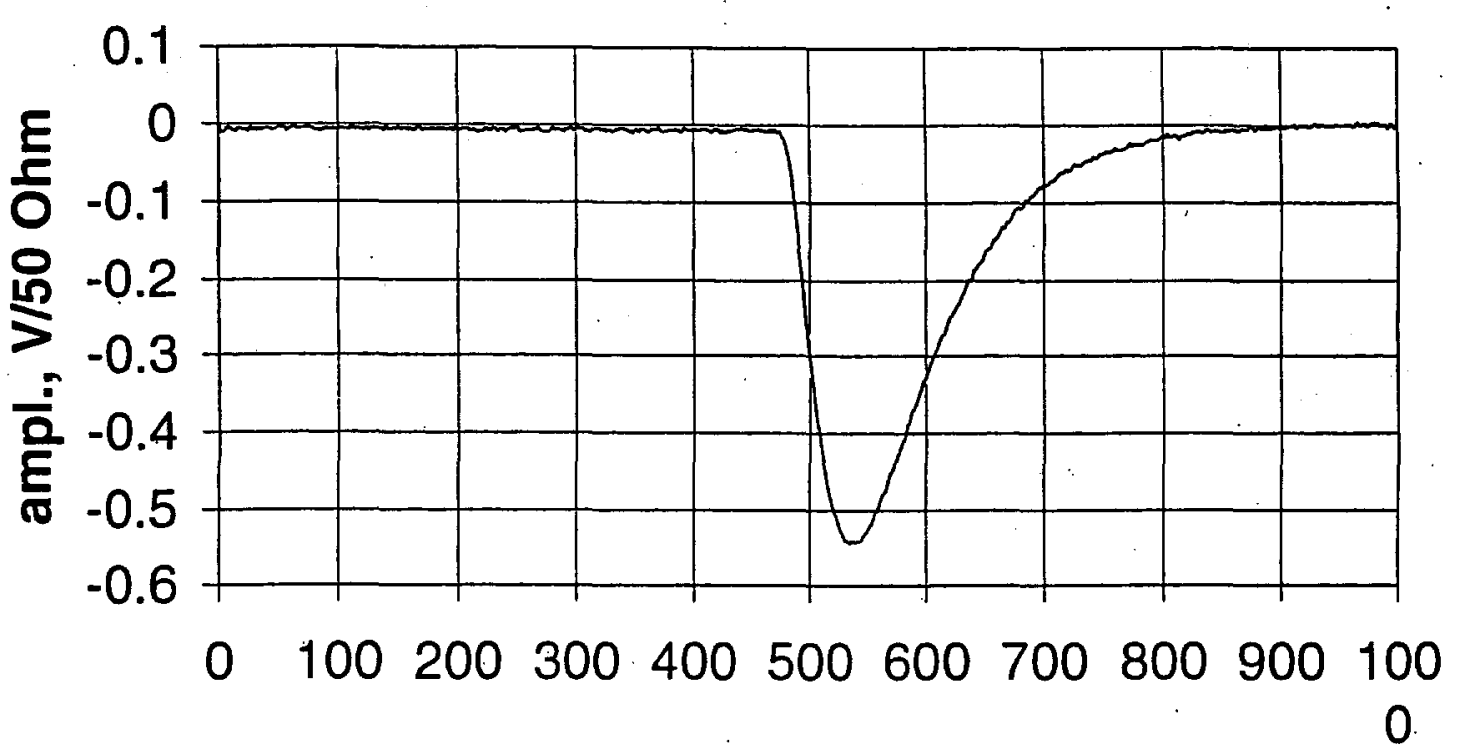

time, ns

Fig.7

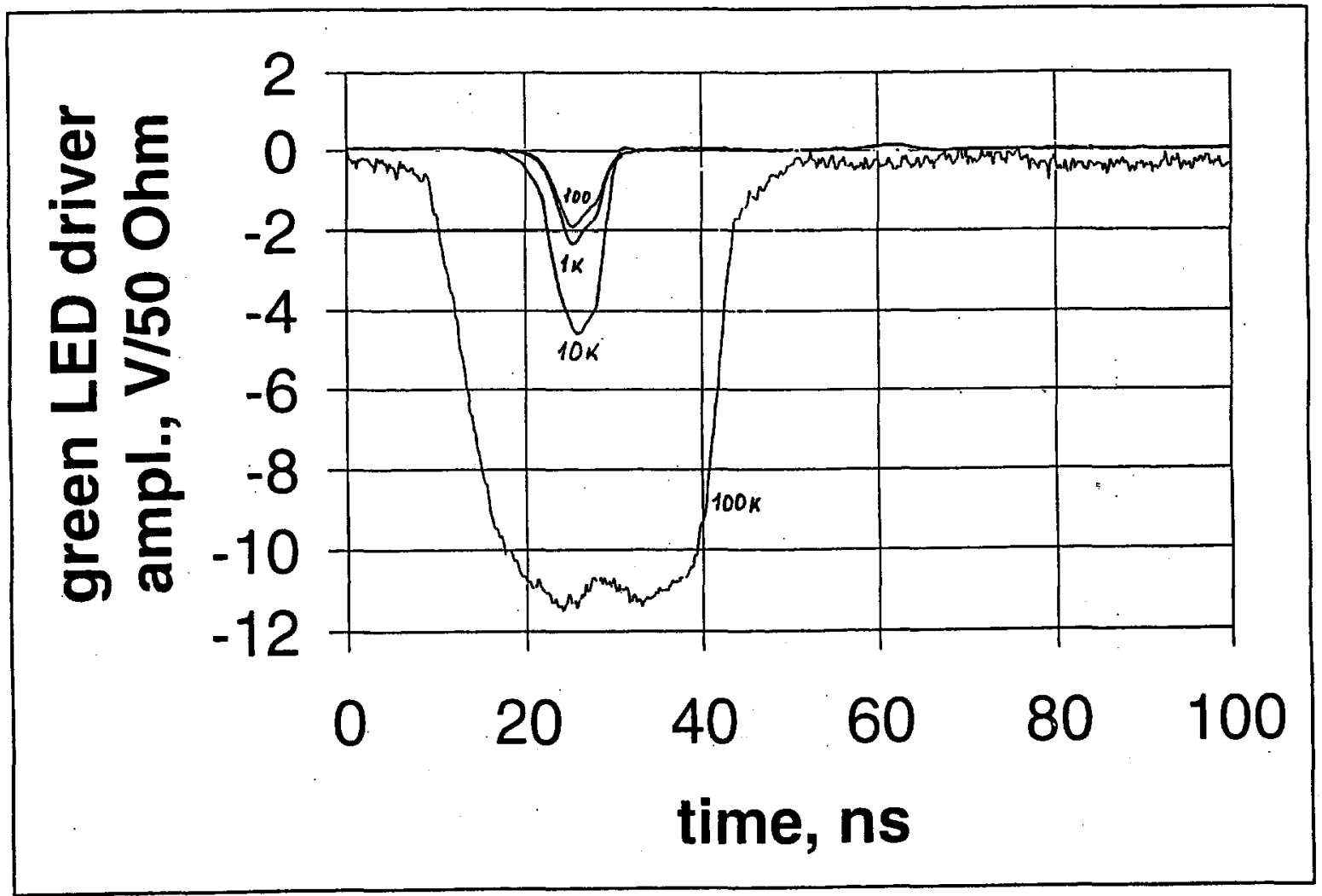

Fig.8 


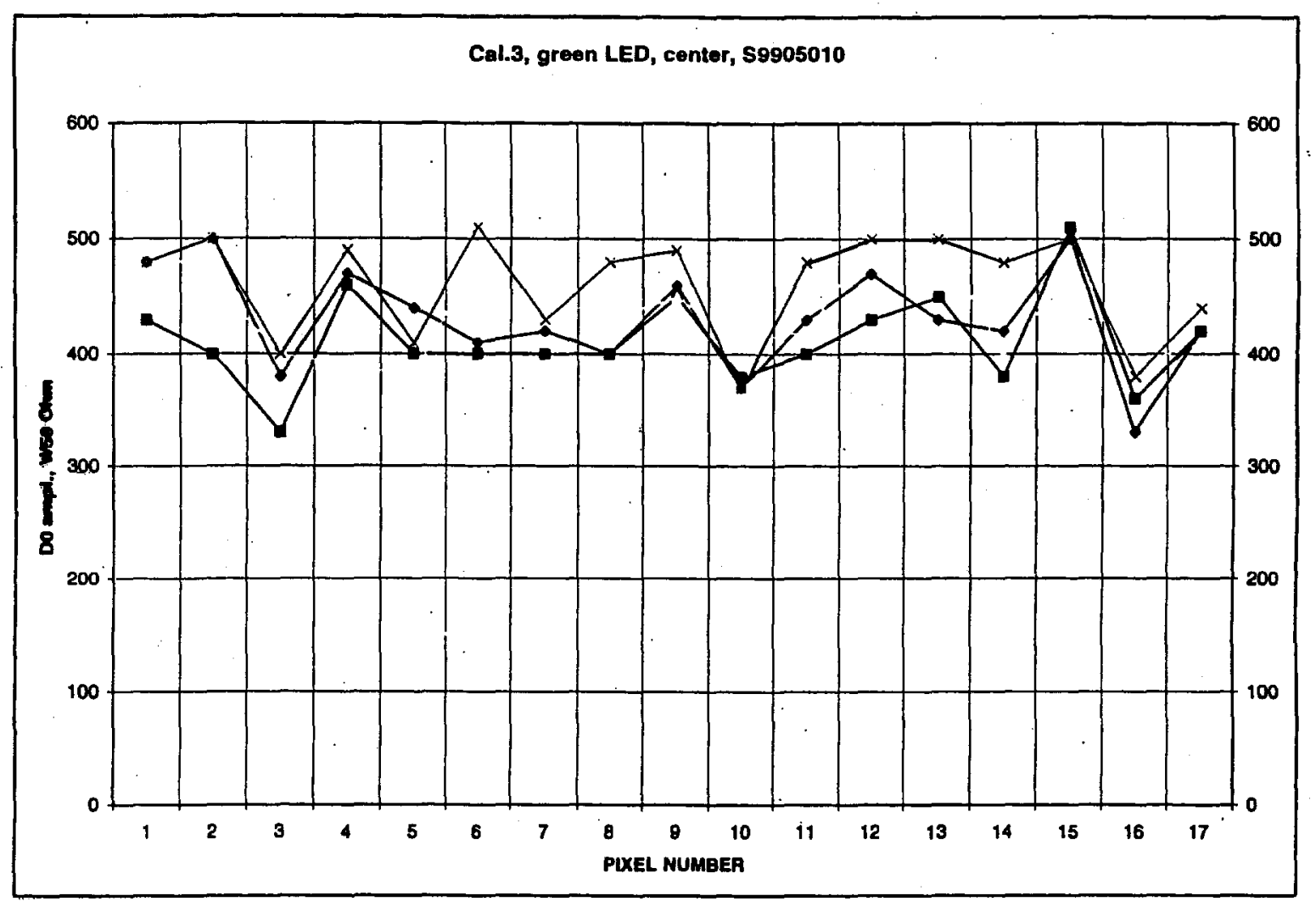

Fig.9

\section{cal.3, green LED, S9905010 (pix $10,10 \mathrm{kV}, 100 \mathrm{~V}$}

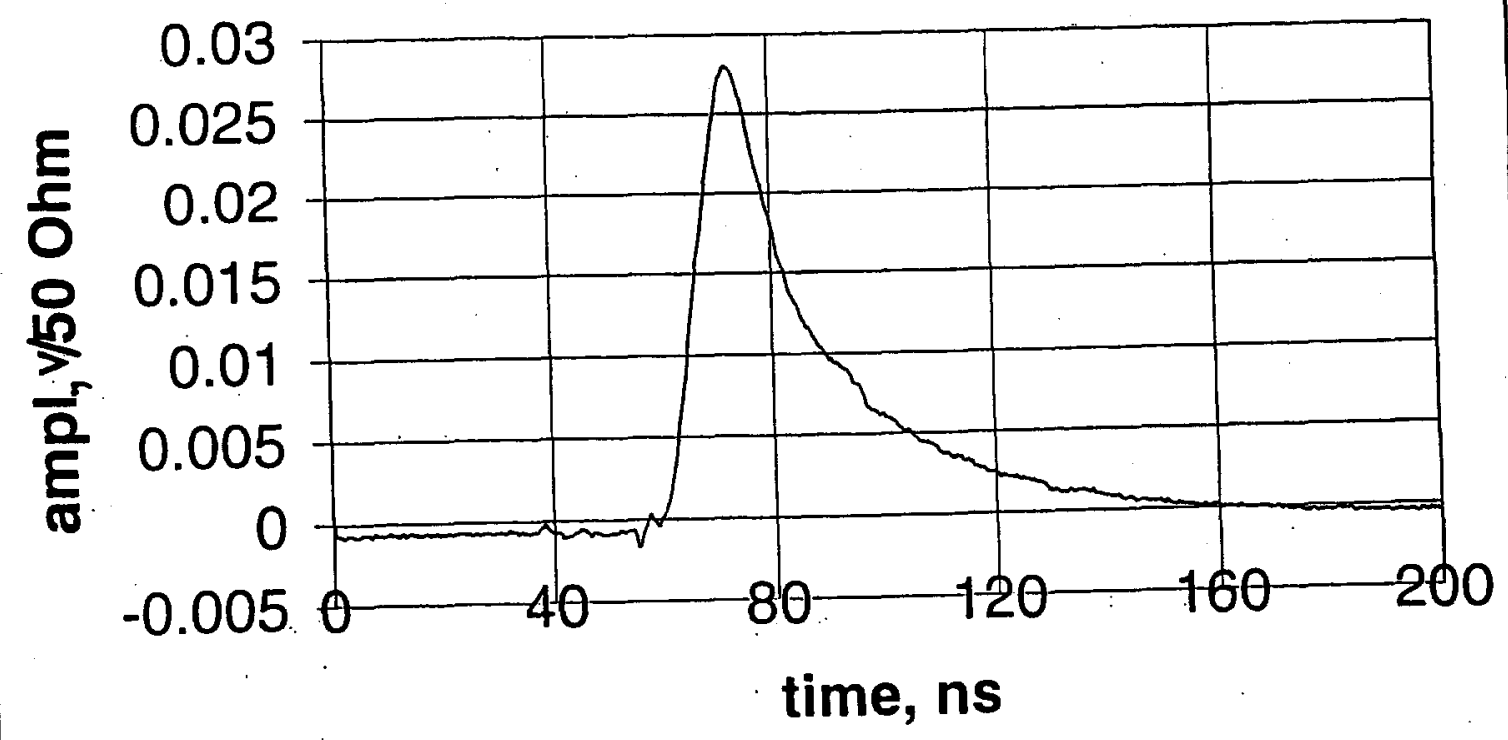

Fig.10 


\section{laser, cal.3 \& SCSN81}

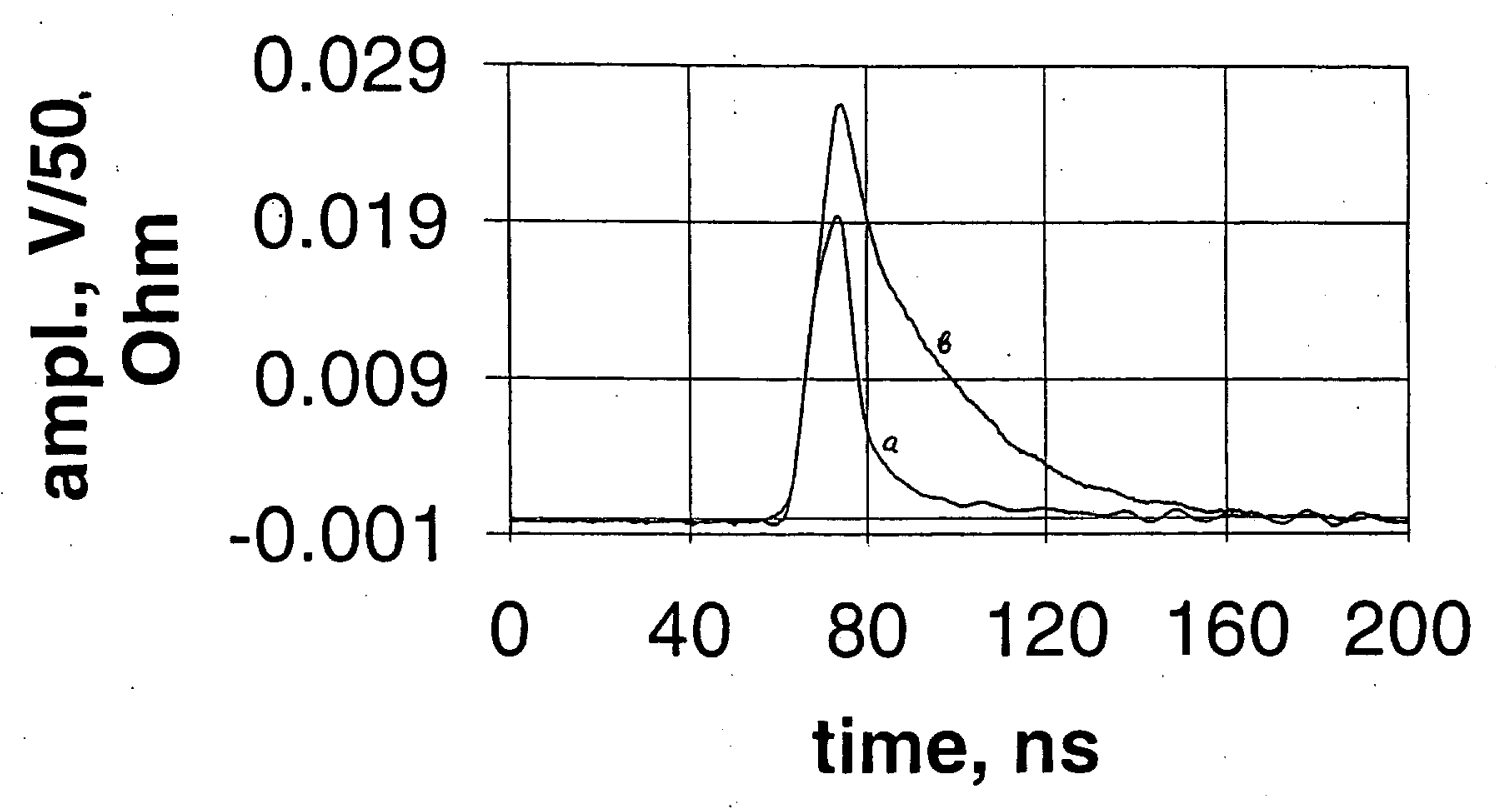

Fig.11

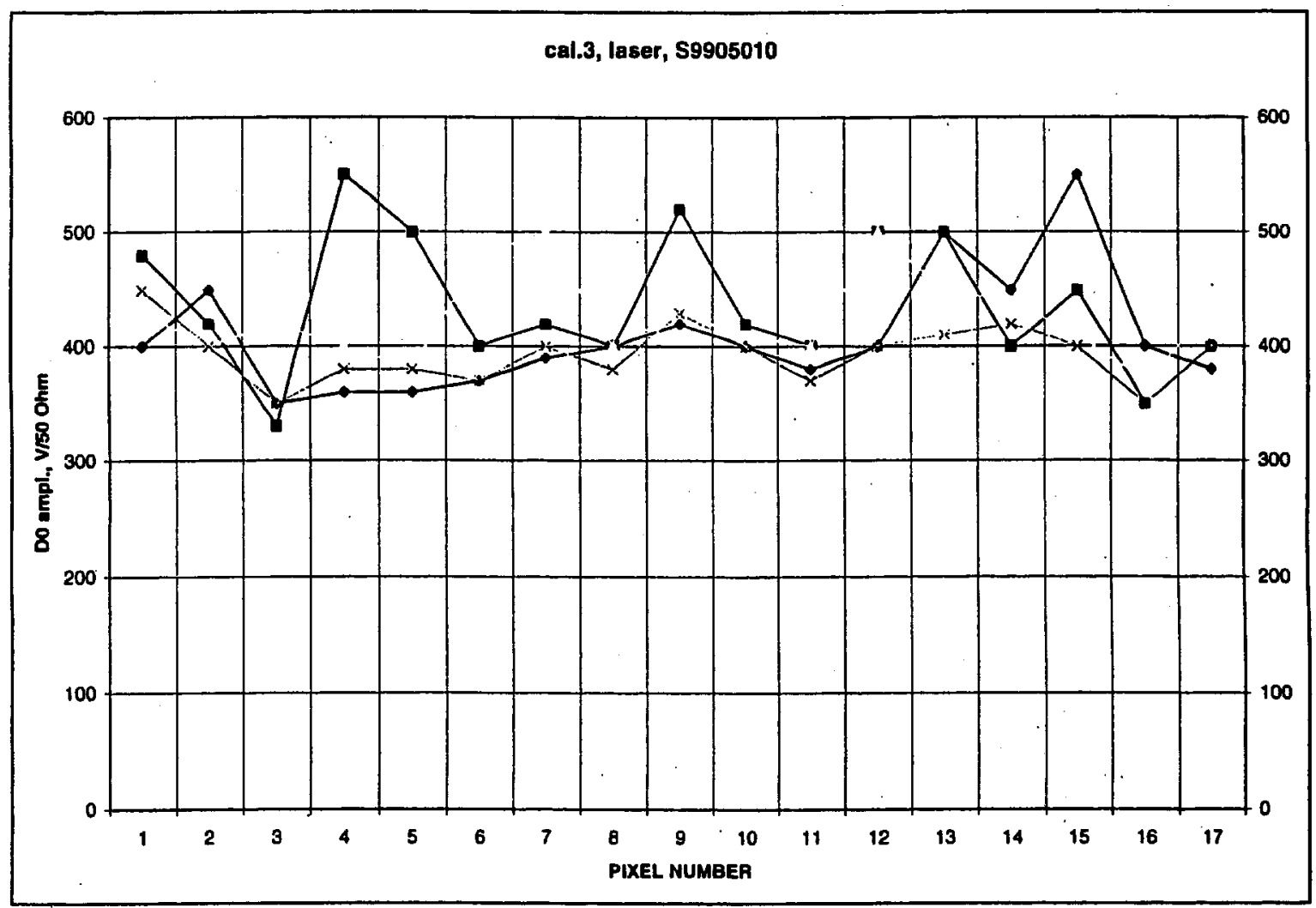

Fig.12 


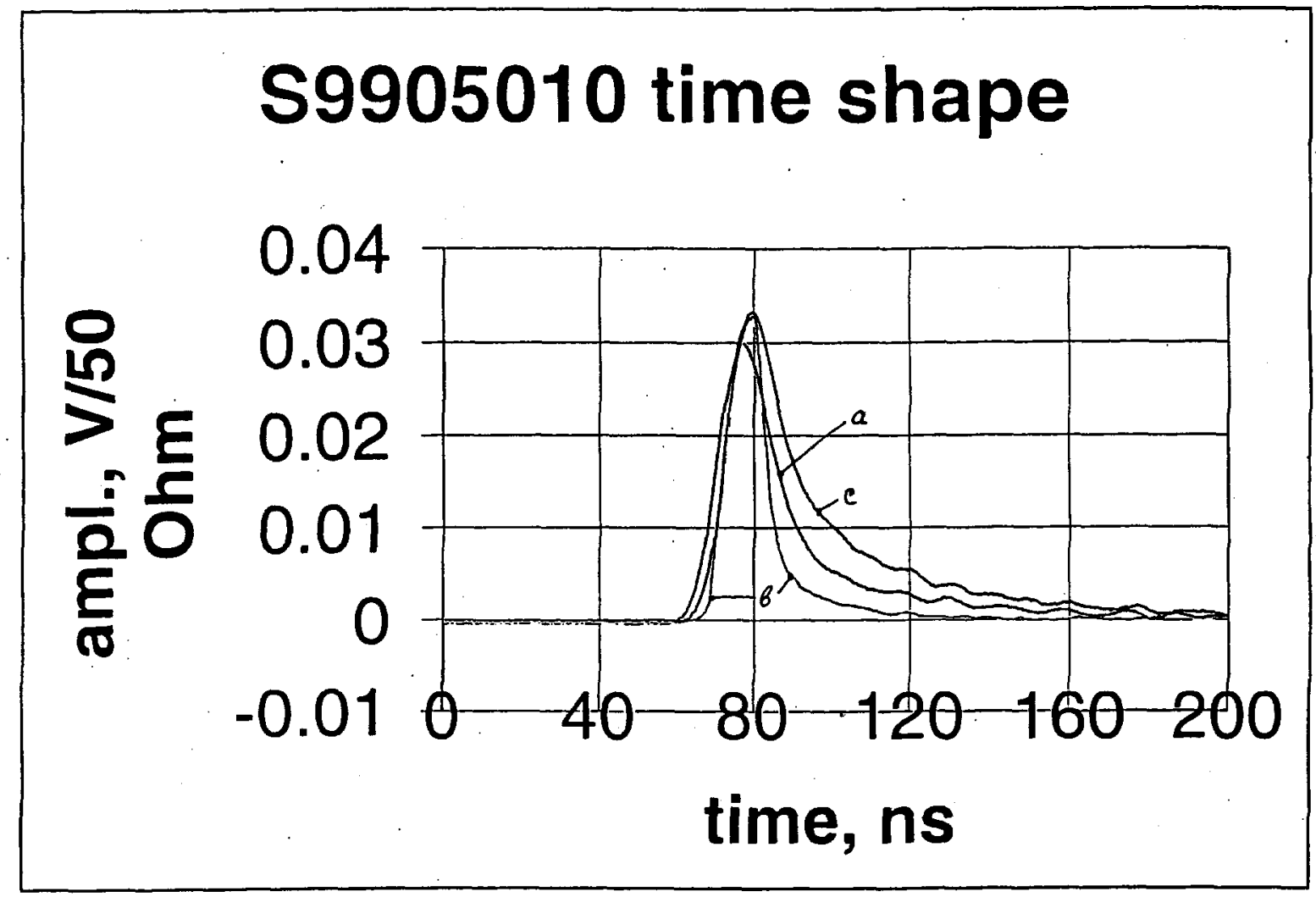

Fig.13

\section{$337 \mathrm{~nm} ; 5 \mathrm{~m}$ q.f; $30 \mathrm{~mm}$ SCSN81; S9905010, $10 \mathrm{kV}$, bias 50-100V}

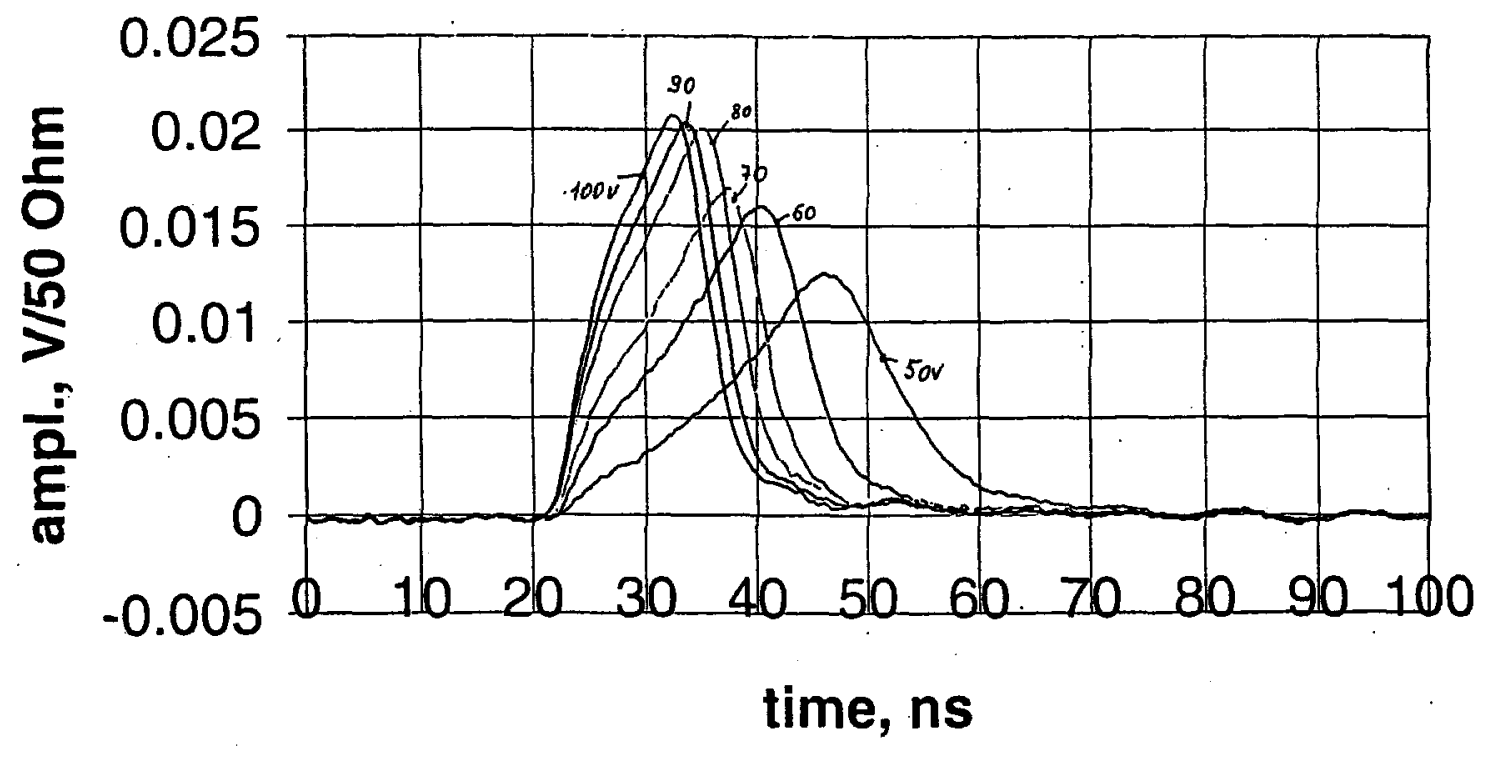

Fig.14 